\title{
Gallium Nitride Porous Microtubules Self-Assembled from Wurtzite Nanorods
}

\author{
Yucheng Lan ${ }^{\mathrm{a}, \mathrm{b}, *}$, Feng $\operatorname{Lin}^{\mathrm{c}, * *}$, Yang $\mathrm{Li}^{\mathrm{d}}$, Yasmin Dias ${ }^{\mathrm{a}}$, Hui Wang ${ }^{\mathrm{b}}$, Yuan \\ $\mathrm{Liu}^{\mathrm{b}}$, Zhen Yang ${ }^{\mathrm{b}}$, Haiqing Zhou ${ }^{\mathrm{b}}$, Yalin $\mathrm{Lu}^{\mathrm{e}}$, Jiming Bao ${ }^{\mathrm{d}}$, Zhifeng Ren ${ }^{\mathrm{b}}$, \\ Martin A. Crimp ${ }^{f}$ \\ ${ }^{a}$ Department of Physics and Engineering Physics, Morgan State University, Baltimore, MD \\ 21251 \\ ${ }^{b}$ Department of Physics and Texas Center for Superconductivity, University of Houston, \\ Houston, TX 77024 \\ ${ }^{c}$ School of Physics, State Key Laboratory for Mesoscopic Physics, Peking University, \\ Beijing 100871, China \\ ${ }^{d}$ Department of Electrical and Computer Engineering, University of Houston, Houston, TX \\ 77204, USA \\ ${ }^{e}$ Laser Optics Research Center, US Air Force Academy, Colorado Springs, CO 80840 \\ ${ }^{f}$ Department of Chemical Engineering and Material Sciences, East Lansing, MI 48824
}

\begin{abstract}
Wurtzite gallium nitride microscale tubules were synthesized through chemical vapor deposition method. The tubules were self-assembled from nano rods without any templates. The self-assemblies were up to one hundred micrometers long and ten micrometers in diameter. The tubules were porous. The unique structures of the tubules caused red-shifts of Raman modes and yellow band in PL spectra. A possible growth mechanism of the porous microtubules was proposed based on experimental observations.
\end{abstract}

Keywords: B2. Semiconducting III-V materials, A1. Low dimensional structures, A1. Nanostructures, A1. Growth models, A1. Crystal morphology

\section{Introduction}

For decades, scientists have been interested in self-assemblies of nanomaterials. Since the discovery of $\mathrm{C}_{60}$ [1] and carbon nanotubes [2], various nanoparticles [3], nanowires [4], and nanotubes [5] etc. have been synthesized. These

\footnotetext{
*Corresponding author: yucheng.lan@morgan.edu

${ }^{* *}$ Corresponding author: linf@pku.edu.cn
}

Preprint submitted to Journal of Crystal Growth

January 6, 2015 
5 nanoscaled materials can self-assemble into various macroscopic structures [6], such as carbon nanotube micro-rings $[7,8]$, gallium nitride nanocrystal-assembled bulks in millimeters [9], and thermoelectric bulk nanocomposites in inches [10, 11], without any templates. Among the self-assembled macroscopic structures, microtubular structures consisting of nanoparticles are expected to facilitate wide applications in electrochemistry [12, 13], biosensors [14] and field emission [15]. These macroscopic structures possess high surface area and quantum confinement effects associated with their constituent nanomaterials, resulting in superior properties over three-dimensional bulk nanocomposites.

However, it is challenging to synthesize microtubular structures with quantum effects. Various microscaled hollow tubular structures have been synthesized without templates, such as element microtubes (gold microtubes [16], selenium microtubes [17], tellurium microtubes [18, 19], silicon microtubes [20], and carbon microtubes [21]), surfide microtubes (ZnS [22], $\mathrm{MoS}_{2}$ [23], and $\mathrm{PbS}[24]$ ), oxides microtubes ( $\mathrm{ZnO}[25-27]$ and $\mathrm{Al}_{18} \mathrm{~B}_{4} \mathrm{O}_{33}[28]$ ), borate microtubes (AlB

20 [29]), pharmaceutical microtubes [30], and organic microtubes [31-33]. The crystalline size of the constituent nanostructures, as well as the walls of these microtubes, were several hundred nanometers to several microns in diameter. Therefore, these microtubes show only limited quantum properties. To date, it is still very challenging to self-assemble zero-dimensional nanoparticles, one25 dimensional nanowires, or one-dimensional nanotubes into three-dimensional ordered structures to achieve macroscaled tubular materials with quantum confinement effects.

Gallium nitride (GaN) semiconductors have a direct band-gap of $3.4 \mathrm{eV}$ and have been widely applied in blue and UV light emission devices and laser devices so $[34,35]$. To date, many kinds of GaN nanomaterials, including GaN nanoparticles [36], GaN nanorods [37, 38], GaN nanowires [39-42], GaN nanotubes [43-46], and GaN nanocomposites [9] have been synthesized. GaN square microtubes were recently reported using a thermal vapor deposition method [47]. Such square microtubes consisted of zinc-blende GaN nanoparticles. Here, an35 other kind of GaN microscaled tubes is presented. The presented microtubes 
are circular and consisted of wurtzite GaN nanorods. The self-assembly growth mechanism is proposed based on experimental phenomena.

\section{Experimental}

The synthesis procedures of microtubules are carried out in a hot-walled chemical vapor deposition (CVD) system consisted of a horizontal quartz tube, a resistance tube furnace equipped with a temperature controller, and a molecular pump backed by a mechanical pump. Type- $K$ thermocouples are used to monitor and control temperatures of the CVD system. The gallium metal (99.999 \% purity) was purchased from the Sigma-Aldrich Corporation. Poly-

45 crystalline quartz substrates are washed by ultrasonicating in acetone and deionized water, followed by thorough rinsing with methanol and drying in air. Gallium is placed on the quartz substrates and located at the center of the horizontal quartz tube. Then the reaction system is evacuated by the molecule pump backed by the mechanic pump, and purged / rinsed several times by argon 50 gas before introducing ammonia gas. The gallium / substrates are then heated under a constant flow of argon gas. The temperature gradually increases from room temperature to $820-840{ }^{\circ} \mathrm{C}$ over 20 minutes. The flowing argon gas is then switched to ammonia gas. The gallium / substrates are maintained at the reaction temperature and kept under the flowing ammonia gas for two minutes.

55 As-received analytical grade commercial argon gas and ammonia gas $(99.9 \%$ purity) are used without further purification throughout the procedures. Afterwards, the flow of ammonia is stopped and the entire system is rapidly cooled to room temperature.

X-ray diffraction (XRD) data is collected on a Sintag XDS $2000 \theta$-2 $\theta$ diffractometer (Thermo Fisher Scientific Inc.) using a step mode of $0.02^{\circ}$ with $5 \mathrm{~s}$ per step, operating at $45 \mathrm{kV}$ voltage and $40 \mathrm{~mA}$ current with graphite-monochromatized $\mathrm{Cu}-K_{\alpha}$ X-ray radiation of $\lambda=1.5415 \AA$. Scanning electron microscopy (SEM) images are taken in secondary electron imaging mode using a CamScan FE 44 SEM (CamScan Electron Optics Ltd., U. K.) operated at 25 kV. Transmission 


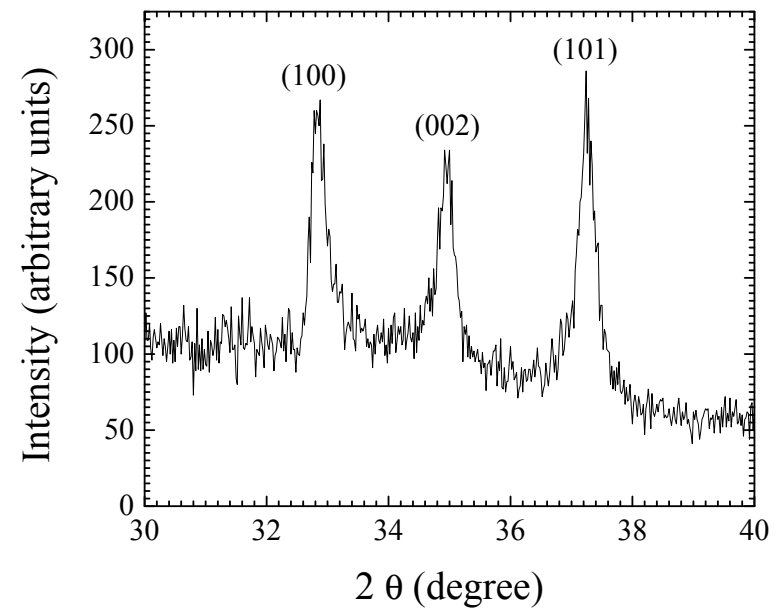

Figure 1: XRD pattern of the as-prepared GaN microtubules. (JEOL Ltd., Japan).

Raman spectra are collected on a WITec CRM200 confocal Raman system (WITec Corp., Germany). An excitation laser with $\lambda=532 \mathrm{~nm}$ is focused onto individual porous GaN microtubules using a $100 \times$ optical lens (numerical aperture NA $=0.95)$. The laser spot is about $500 \mathrm{~nm}$ in diameter. The Raman signals are routed to a TE-cooled charge-coupled-device cooled to $-60{ }^{\circ} \mathrm{C}$.

The micro-PL measurement on the GaN microtubes is performed in a confocal configuration at room temperature using a $532 \mathrm{~nm}$ laser as the excitation source, with the power of $0.4 \mathrm{~mW}$. The spectra are obtained by using a Horiba 75 Triax550 spectrometer equipped with a thermo-electrically cooled charge coupled device. The laser beam is focused on individual GaN microtubules with a $50 \times$ objective under an optical microscope when the PL spectra are collected.

\section{Results and Discussion}

GaN Microtubules. After the synthesis procedures, yellow layers are deposited 80 

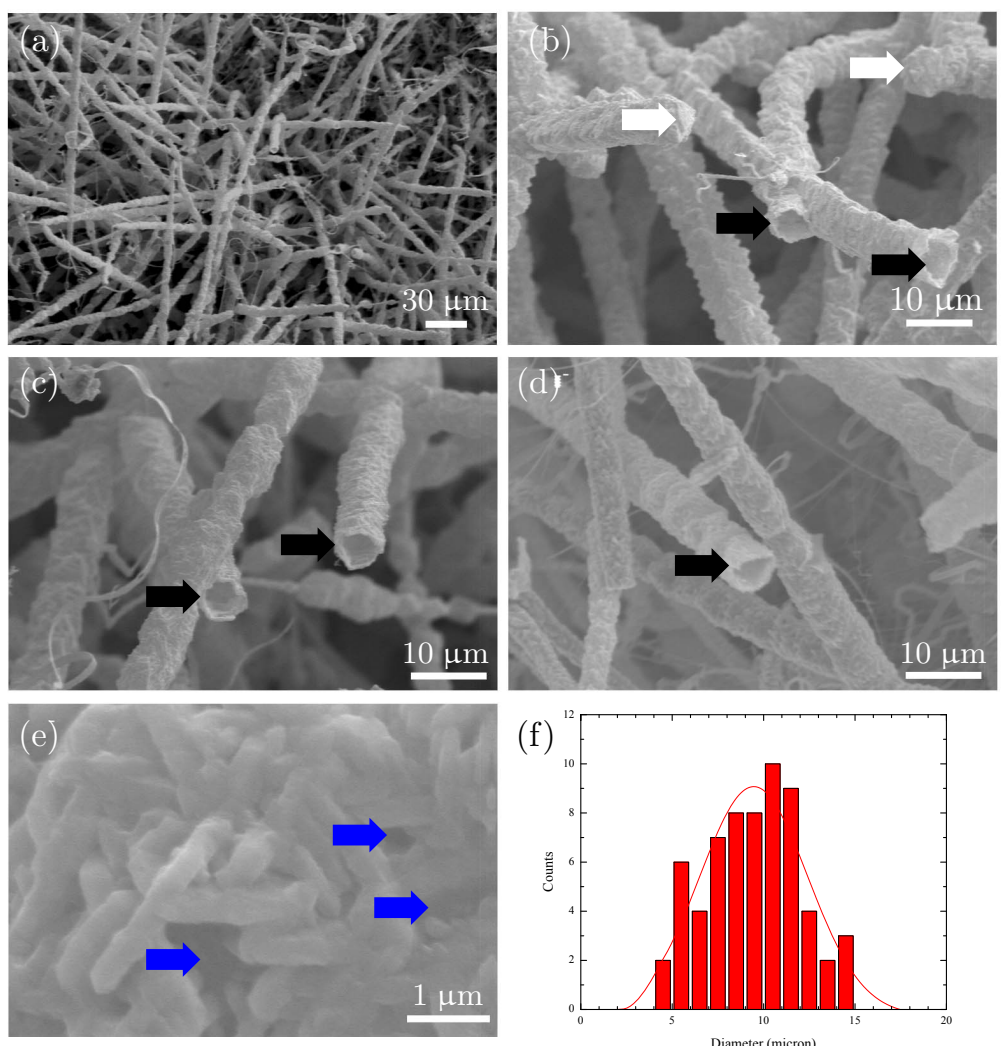

Figure 2: SEM images of (a-d) microtubules grown at different regions and (e) wall surface of a microtubule. White arrows mark the closed ends, black arrows the open ends, and the blue arrows the nanopores of the microtubules. (f) Histogram of microtubule diameter.

rectly from the yellow layers deposited on the substrates. Figure 1 shows a typical XRD pattern of the yellow layers. All of the broadened XRD peaks can be indexed on the basis of the hexagonal wurtzite GaN phase with a space group of $\mathrm{P} 6_{3} \mathrm{mc}$ [48]. The broadening of the XRD peaks possibly come from defects or crystalline size of the synthesized materials. The internal strain is calculated using the Williamson-Hall method from the XRD data, $\epsilon=0.0022$. The internal stress of the produced materials can be calculated if we assume that the produced nanomaterials are spherical. The calculated internal stress of the synthesized materials is $\sigma=0.46 \mathrm{GPa}$.

Figure 2a-d show SEM images of the yellow products on the substrates. 
The synthesized structures are hollow. The cross-section of the microtubules is circular. The ends of most of the microtubules are open (marked with black arrows) while some are closed (marked with white arrows). The length of the microtubes is up to $100 \mu \mathrm{m}$.

Eighty-five microtubules are randomly chosen from ten different regions of the substrate surfaces (some are shown in Figure 2a-d) and their diameters are measured from SEM images. The microtubules grown on the same region are uniform while those grown on different regions are slightly different, especial in diameters. Figure $2 \mathrm{f}$ plots the diameter distribution of these microtubules. The statistical analysis indicates that the diameter of the synthesized microtubules ranges from $4 \mu \mathrm{m}$ to $15 \mu \mathrm{m}$ with an average value of $10 \mu \mathrm{m}$.

By observing the open ends of the microtubules, the thickness of the microtubular walls is measured from fifteen microtubules, and is found to be $0.13-0.52 \mu \mathrm{m}$ with an average of $0.24 \mu \mathrm{m}$.

High magnification SEM images (i. e., Figure 2e) indicate that the walls of the microtubules consist of short nanorods. Most of the nanorods are $2 \mu \mathrm{m}$ long and about $0.3 \mu \mathrm{m}$ in diameter. Consequently, the wall is essentially 1-2 nanorods thick, highly defective with many nanopores (as marked with blue arrows in Figure 2e), and have very large surface areas.

As expected, the porous microtubules are brittle. While in some instances the microtubules are picked up carefully using metal tweezers under an optical microscope, most of the microtubules are broken when they are transferred from the substrates to TEM grids. This is especially noticeable for longer microtubules with large diameters and thin walls. Only shorter microtubules with thicker walls and smaller diameter can be transferred to TEM grids.

Figure 3a shows a typical bright-field TEM (BF-TEM) image of an intact microtubule on a TEM grid. The diameter is about $5 \mu \mathrm{m}$ and the wall thickness, as suggested by the region of dark contrast at the outer edges of the tubule, is near $0.5 \mu \mathrm{m}$. The microtubule is in-situ rotated around its axis from $-15^{\circ}$ to $15^{\circ}$ on TEM. The width of the microtubule and the darker edges are independent on the rotation. The wall thickness ranges from $0.2 \mu \mathrm{m}$ to $0.5 \mu \mathrm{m}$. Therefore 

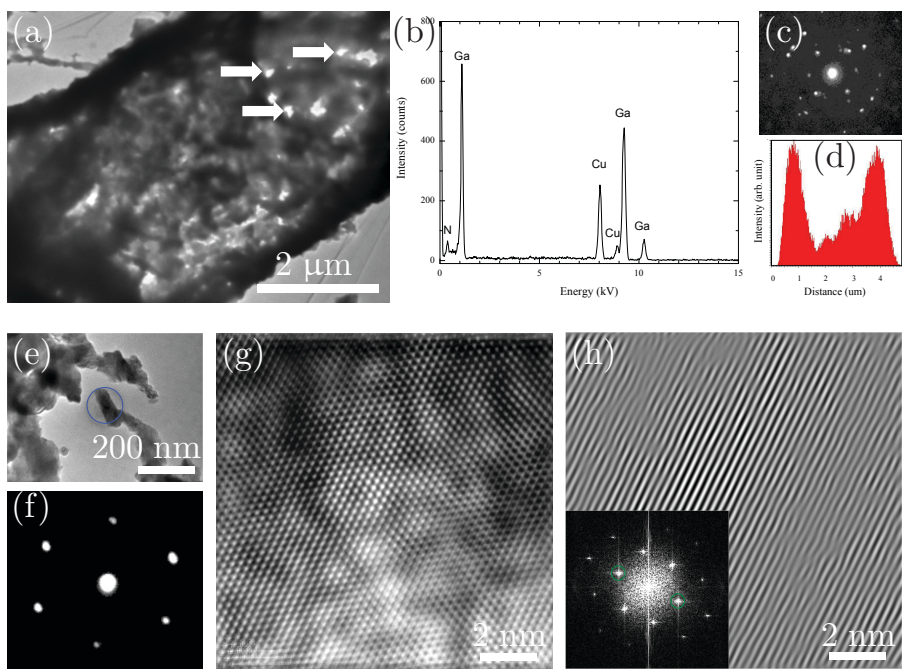

Figure 3: (a) BF-TEM image of a GaN microtubule. Several typical nanopores are marked by arrows. (b) EDS spectrum of the microtubule in (a). Cu signal coming from the TEM grid. (c) SAED pattern of the microtubule in (a). (d) EDS linescan of Ga- $K_{\alpha}$ across the microtubule in (a). (e) BF-TEM image of several adjacent nanorods near a nanopore. (f) SAED of the nanorod circled in (e). (g) HRTEM image of the nanorod circled in (e). (h) IFFT of (g). Inset: FFT of (g). Two green circles mark the diffraction spots used for IFFT. 
the microtubule is in fact tubular hollow structure with a nano-wall.

TEM images confirm that the microtubules are porous besides SEM observations. Consequently, the walls display significant roughness. The intensity variations observed across the images are consistent with large variations in the grey density of the walls of the microtubule, with some nanopores marked with the arrows in Figure 3a. Electron X-ray dispersive spectroscopy (EDS), shown in Figure $3 \mathrm{~b}$, indicates that the microtubule is composed of gallium and nitrogen. The chemical composition is in agreement with the XRD data indicating the crystal structure is consistent with that of GaN. Selected area electron diffraction (SAED) patterns, collected from the microtubule and shown in Figure 3c, indicates that the microtubule is polycrystalline.

Figure $3 \mathrm{~d}$ shows EDS gallium $\mathrm{K}_{\alpha}$ intensity across the microtubule. The EDS linescanning indicates that the microstructure is hollow, with a stronger EDS signal near the walls of the microtubule.

Figure 3e shows several adjacent nanorods near a nanopore. These nanorods are overlapped and connected to each other. One nanorod is chosen (marked with a blue circle) to study crystallinity. Figure 3 f shows its SAED pattern. The SAED indicates that the individual nanorod is a single crystal. High-resolution TEM (HRTEM) images of the nanorod are taken at room temperature and Figure $3 \mathrm{~g}$ shows the core region of the nanorod. Although the nanorod is crystalline, there is a lot of atomic distortion in the lattice, as shown in Figure $3 \mathrm{~h}$ which is the inverse fast Fourier transform (IFFT) of Figure 3g along a certain direction. From the core to the edge of the nanorod, the crystallinity is worse and worse. More atomic distortions and dislocations are observed in the edge regions of the nanorod.

Raman scattering. Microstructures of the nanorods affect optical properties of the microtubules. Figure 4 shows a typical Raman spectrum of a GaN microtubule measured at room temperature. The laser beam is focused on one GaN microtube under an optical microscope and the Raman spectrum is collected from the individual microtubule. Wurtzite $\mathrm{GaN}$ with $\mathrm{P} 6{ }_{3} \mathrm{mc}$ space group 


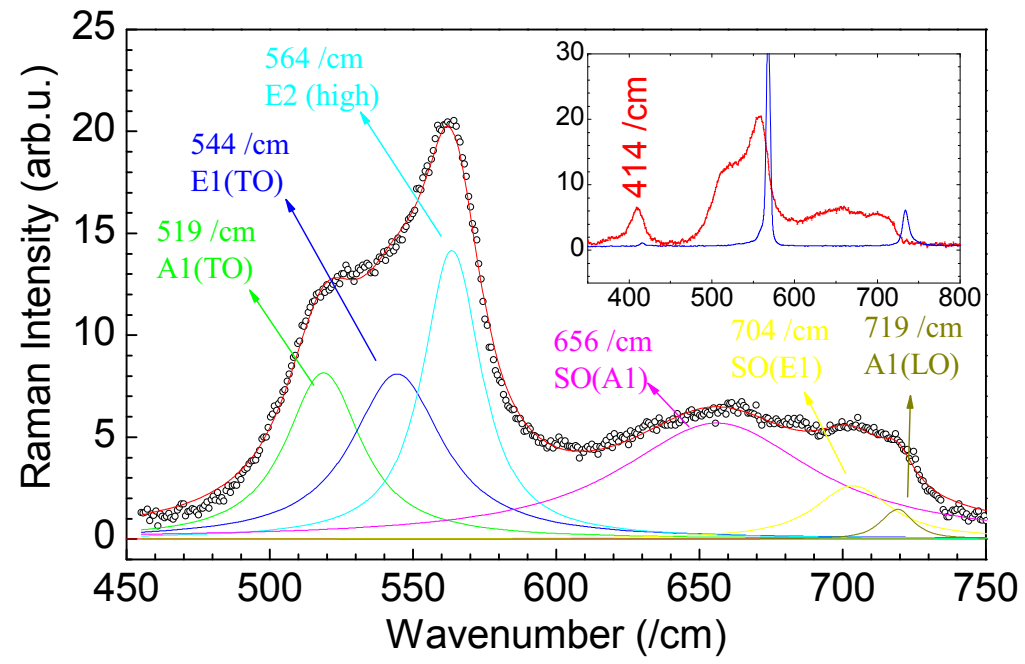

Figure 4: Raman scattering of a GaN microtubule. o: experimental; - : fitting. Inset: complete range of Raman scattering of the microtubule (red curve) compared with that of GaN crystalline films (blue curve).

has six active Raman modes: $\mathrm{A}_{1}(\mathrm{TO})+\mathrm{A}_{1}(\mathrm{LO})+\mathrm{E}_{1}(\mathrm{TO})+\mathrm{E}_{1}(\mathrm{LO})+2 \mathrm{E}_{2}$ [49]. Four active modes are observed here. The peaks at 519, 544, 564, and $719 \mathrm{~cm}^{-1}$ correspond to the $\mathrm{A}_{1}(\mathrm{TO}), \mathrm{E}_{1}(\mathrm{TO}), \mathrm{E}_{2}$ (high), and $\mathrm{A}_{1}(\mathrm{LO})$ symmetries, respectively. Compared with bulk data $\left(532 \mathrm{~cm}^{-1}\right.$ for $\mathrm{A}_{1}(\mathrm{TO}), 559 \mathrm{~cm}^{-1}$ for $\mathrm{E}_{1}(\mathrm{TO}), 568 \mathrm{~cm}^{-1}$ for $\mathrm{E}_{2}$ (high), $734 \mathrm{~cm}^{-1}$ for $\left.\mathrm{A}_{1}(\mathrm{LO})\right)$ [49], the four active Raman modes significantly red shift to shorter wavenumbers.

Two other Raman modes are observed on the low wavenumber side of the $\mathrm{A}_{1}(\mathrm{LO})$ mode. The two peaks at $656 \mathrm{~cm}^{-1}$ and $704 \mathrm{~cm}^{-1}$ should be surface optical (SO) phonon modes corresponding to $\mathrm{A}_{1}$ and $\mathrm{E}_{1}$ symmetries and caused by surface effects of the GaN nanorods.

A unusual Raman peak is observed at $414 \mathrm{~cm}^{-1}$ (inset of Figure 4). The peak should be the acoustic overtone [50] from wurtzite GaN nanorods.

PL spectrum. Figure 5a shows a typical photoluminescence (PL) spectrum of 165 an individual GaN circular microtube, excited by $532 \mathrm{~nm}$ wavelength radiation. The laser beam is focused on the individual microtube under an optical micro- 

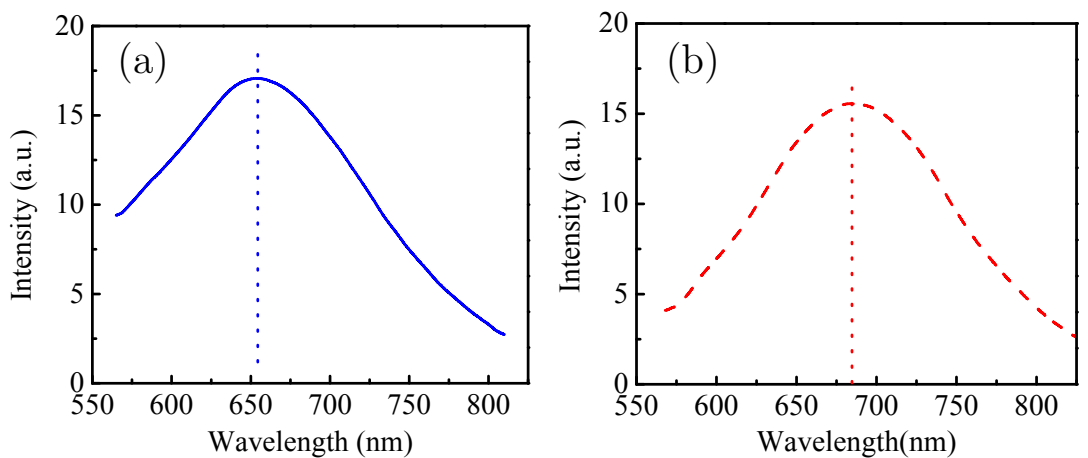

Figure 5: Photoluminescence spectra of (a) a GaN circular microtube with wurtzite structure and (b) a GaN squared microtube with zinc-blende structure.

scope to excite the PL spectrum. The collected PL signals should only come from the circular microtubule, not from other nanomaterials underneat the microtubule. A yellow band, centered at $653 \mathrm{~nm}$, is observed. Similar yellow bands were also observed in GaN nanocomposites [9], nanoparticles [36], and nanowires [39] with wurtzite structure.

A PL spectrum of zinc-blende microtubes [47] is shown in Figure 5b for comparion. The PL spectrum of the zinc-blende microtubes is centered at $685 \mathrm{~nm}$. The PL spectrum of the circular microtubes blue-shifts to higher energy, reflecting the fact that the wurtzite GaN has a wider band-gap than the cubic GaN $[50]$.

Growth Mechanism. The formation of the porous microtubules can be explained by the Liesegang phenomenon [51] and diffusion limited growth. Gallium has a low melting point of $303 \mathrm{~K}$ and a boiling point of $2520 \mathrm{~K}$. Therefore, large amounts of gallium liquid droplets can condense on quartz substrates from gaseous gallium (Figure 6a), as observed on silicon substrates [52]. The diameter of the condensed droplets is usually $5-50 \mu \mathrm{m}$ [53], depending on reaction temperatures and substrates. The behavior of gallium is very similar to that of other low melting point metals, such as selenium [17]. Gallium droplets evaporate at high temperatures to produce gallium vapor during the CVD procedure. 
(a)

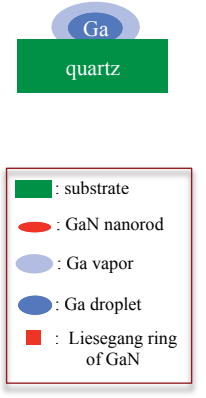

Initial Stage (b)
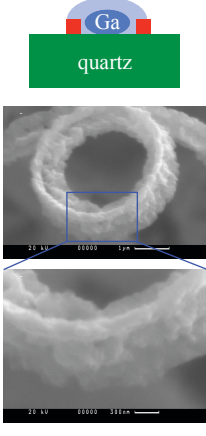

Template Stage (c)
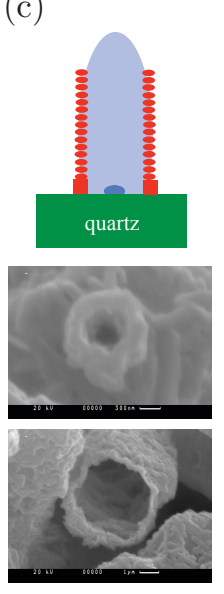

Growth Stage (d)

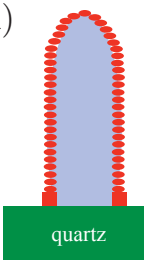

top

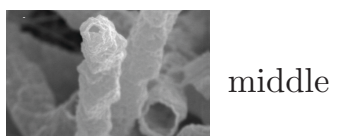

bottom

Enclosing Stage

Figure 6: Possible growth mechanism of the self-assembled GaN microtubules. (a) Initial stage. No GaN nanomaterials are synthesized. (b) Template stage. Liesegang rings of GaN nanomaterials are formed. (c) Growth stage. GaN nanorods self-assemble into microtubules. (d) Enclosing stage. Ga sources are exhausted and the ends of GaN microtubules are closed. Top: model of each stage. Middle and bottom: SEM images at each stage.

Once ammonia passes the gallium droplets, gallium vapor would react with ammonia to produce GaN nanoparticles (Figure 6b). The number of the GaN nanoparticles per unit volume is so dense around the gallium droplets that the GaN nanoparticles around the gallium droplets can be considered as a supersaturated aerosol, similar to charged nanoparticles in solutions [54] and nanoparticles in vapor phases $[55,56]$. These supersaturated GaN nanoparticles would spontaneously aggregate into Liesegang rings due to thermodynamatic stability, as observed in other kinds of ordered Liesegang nanoparticle-rings [54, 57].

The proposed growth mechanism is experimentally confirmed by SEM observation. The middle panel in Figure 6b shows an SEM image of a typical GaN nanoparticle Liesegang ring with a diameter of several micrometers. A higher magnification SEM image (bottom panel in Figure 6b) indicates that the microring consists of many GaN nanoparticles with a size below one hundred nanometers. Other size GaN rings are also observed on the substrates. These surviving 

tics of super-saturation growth caused by the Liesegang effect. 


\section{Conclusion}

One kind of novel gallium nitride circular tubules are grown from wurtzite

230 porous, several tens of micrometers long and several microns in diameter. The constituent nanorods are crystalline with lots of defects. The self-assembly of GaN nanomaterials is contributed to the diffusion limited growth of supersaturated GaN nanorods. Compared with bulk crystals, the Raman active modes of 235 the microscale tubules red shift. The red-shift should come from the defects in the microstructure. A yellow band was observed in photoluminescence spectra of the grown nanomaterials.

\section{Acknowledgements}

The authors YCL and ZFR thank the financial support by the Defense 240 support from US Department of Energy under the contract DE-NA0000720 through the program of S.P. Massie Chair of Excellence, the Small Grant Program from the University of Houston and the Center for Nanoscale Systems at Harvard University which is supported under NSF ECS-0335765. FL is supported by the National Basic Research Program of China (973 program), Grant No. 2012CB933004. JMB acknowledges the support from the National Science Foundation (CAREER Award ECCS-1240510 and DMR-0907336) and from the Robert A Welch Foundation (E-1728). MAC thanks the Center for Advanced Microscopy at Michigan State University for utilizing the JEM 2200FS electron 250 microscope.

\section{References}

[1] H. W. Kroto, J. R. Heath, S. C. O'Brien, R. F. Curl, R. E. Smalley, $\mathrm{C}_{60}$ : Buckminsterfullerene, Nature 318 (1985) 162 - 163. doi:10.1038/ 318162a0. 
[2] S. Iijima, Helical microtubules of graphitic carbon, Nature 354 (1991) 56 58. doi:10.1038/354056a0.

[3] X. Lu, M. Rycenga, S. E. Skrabalak, B. Wiley, Y. Xia, Chemical synthesis of novel plasmonic nanoparticles, Annu. Rev. Phys. Chem. 60 (1) (2009) 167 - 192. doi:10.1146/annurev.physchem.040808.090434.

[4] W. Lu, C. M. Lieber, Semiconductor nanowires, J. Phys. D: Appl. Phys. 39 (21) (2006) R387. doi:10.1088/0022-3727/39/21/R01.

[5] Y. Lan, Y. Wang, Z. F. Ren, Physics and applications of aligned carbon nanotubes, Adv. Phys. 60 (4) (2011) 553 - 678. doi:10.1080/00018732. 2011.599963.

[6] M. Grzelczak, J. Vermant, E. M. Furst, L. M. Liz-Marzán, Directed selfassembly of nanoparticles, ACS Nano 4 (7) (2010) 3591 - 3605. doi: $10.1021 / \mathrm{nn} 100869 \mathrm{j}$.

[7] R. Martel, H. R. Shea, P. Avouris, Rings of single-walled carbon nanotubes, Nature 398 (1999) 299. doi:10.1038/18589.

[8] M. Sano, A. Kamino, J. Okamura, S. Shinkai, Ring closure of carbon nanotubes, Science 293 (5533) (2001) 1299 - 1301. doi:10.1126/science. 1061050.

[9] X. L. Chen, Y. G. Cao, Y. C. Lan, X. P. Xu, J. Q. Li, K. Q. Lu, P. Z. Jiang, T. Xu, Z. G. Bai, Y. D. Yu, J. K. Liang, Synthesis and structure of nanocrystal-assembled bulk GaN, J. Cryst. Growth 209 (1) (2000) 208 -212. doi:10.1016/S0022-0248(99)00522-9.

[10] B. Poudel, Q. Hao, Y. Ma, Y. Lan, A. Minnich, B. Yu, X. Yan, D. Wang, A. Muto, D. Vashaee, X. Chen, J. Liu, M. S. Dresselhaus, G. Chen, Z. Ren, High-thermoelectric performance of nanostructured bismuth antimony telluride bulk alloys, Science 320 (5876) (2008) 634 - 638. doi: 10.1126/science. 1156446. 
[11] Y. Lan, A. J. Minnich, G. Chen, Z. Ren, Enhancement of thermoelectric figure-of-merit by a bulk nanostructuring approach, Adv. Funct. Mater. 20 (3) (2010) 357 - 376. doi:10.1002/adfm. 200901512.

[12] F. Tao, M. Guan, Y. Zhou, L. Zhang, Z. Xu, J. Chen, Fabrication of nickel hydroxide microtubes with micro- and nano-scale composite structure and improving electrochemical performance, Cryst. Growth Des. 8 (7) (2008) 2157 - 2162. doi:10.1021/cg7012123.

[13] W. Gao, S. Sattayasamitsathit, J. Orozco, J. Wang, Highly efficient catalytic microengines: Template electrosynthesis of polyaniline/platinum microtubes, J. Am. Chem. Soc. 133 (31) (2011) 11862 - 11864. doi: $10.1021 / \mathrm{ja} 203773 \mathrm{~g}$.

[14] T. Fischer, A. Agarwal, H. Hess, A smart dust biosensor powered by kinesin motors, Nat. Nanotech. 4 (2009) 162 -166. doi : 10.1038/nnano.2008. 393.

[15] H. Manohara, W. L. Dang, P. H. Siegel, M. Hoenk, A. Husain, A. Scherer, Field emission testing of carbon nanotubes for $\mathrm{THz}$ frequency vacuum microtube sources, Proc. SPIE 5343, Reliability, Testing, and Characterization of MEMS/MOEMS III 5343 (2004) 227. doi:10.1117/12.531403.

[16] R. Gilliam, S. Thorpe, D. Kirk, A nucleation and growth study of gold nanowires and nanotubes in polymeric membranes, J. Appl. Electrochem. 37 (2007) 233 - 239. doi:10.1007/s10800-006-9240-x.

[17] E. Filippo, D. Manno, A. Serra, Characterization and growth mechanism of selenium microtubes synthesized by a vapor phase deposition route, Cryst. Growth Des. 10 (11) (2010) 4890 - 4897. doi:10.1021/cg1012632.

[18] T. Siciliano, E. Filippo, A. Genga, G. Micocci, M. Siciliano, A. Tepore, Single-crystalline Te microtubes: Synthesis and $\mathrm{NO}_{2}$ gas sensor applications, Sens. Actuat. B: Chem. 142 (1) (2009) 185 - 190. doi:10.1016/j. snb.2009.07.050. 
[19] X.-L. Li, G.-H. Cao, C.-M. Feng, Y.-D. Li, Synthesis and magnetoresistance measurement of tellurium microtubes, J. Mater. Chem. 14 (2004) $244-247$. doi:10.1039/B309097C.

[20] J. Hu, Y. Bando, Z. Liu, J. Zhan, D. Golberg, The first template-free growth of crystalline silicon microtubes, Adv. Funct. Mater. 14 (6) (2004) 610 - 614. doi:10.1002/adfm. 200305138.

[21] J. Libera, Y. Gogotsi, Hydrothermal synthesis of graphite tubes using Ni catalyst, Carbon 39 (9) (2001) 1307 -1318. doi:10.1016/S0008-6223(00) 00263-3.

[22] G. Shen, Y. Bando, D. Golberg, Self-assembled three-dimensional structures of single-crystalline $\mathrm{ZnS}$ submicrotubes formed by coalescence of $\mathrm{ZnS}$ nanowires, Appl. Phys. Lett. 88 (12) (2006) 123107. doi:10.1063/1. 2186980.

[23] M. Remskar, Z. Skraba, F. Cleton, R. Sanjines, F. Levy, $\mathrm{MoS}_{2}$ as microtubes, Appl. Phys. Lett. 69 (3) (1996) 351 - 353. doi:10.1063/1.118057.

[24] W. Wang, Q. Li, M. Li, H. Lin, L. Hong, Growth of PbS microtubes with quadrate cross sections, J. Cryst. Growth 299 (1) (2007) 17 - 21. doi: 10.1016/j.jcrysgro.2006.10.262.

[25] J. Cheng, R. Guo, Q.-M. Wang, Zinc oxide single-crystal microtubes, Appl. Phys. Lett. 85 (22) (2004) 5140 - 5142. doi:10.1063/1.1825067.

[26] T. Sun, J. Qiu, Fabrication of $\mathrm{ZnO}$ microtube arrays via vapor phase growth, Mater. Lett. $62(10-11)$ (2008) 1528 - 1531. doi:10.1016/j. matlet.2007.09.015.

[27] M. Fu, G. Du, X. Fu, B. Liu, Y. Wan, L. Guo, Tetrapod-shaped ZnO microtubes synthesized from Zn/C mixtures, Mater. Res. Bull. 43 (4) (2008) 912 - 918. doi:10.1016/j.materresbull.2007.04.035. 
[28] R. Ma, Y. Bando, T. Sato, C. Tang, F. Xu, Single-crystal $\mathrm{Al}_{18} \mathrm{~B}_{4} \mathrm{O}_{33}$ microtubes, J. Am. Chem. Soc. 124 (36) (2002) 10668 - 10669. doi: 10.1021/ja0271173.

[29] W. Yang, Z. Xie, J. Ma, H. Miao, J. Luo, L. Zhang, L. An, Polygonal singlecrystal aluminum borate microtubes, J. Am. Ceram. Soc. 88 (2) (2005) 485 -487. doi:10.1111/j.1551-2916.2005.00084.x.

[30] M. D. Eddleston, W. Jones, Formation of tubular crystals of pharmaceutical compounds, Cryst. Growth Des. 10 (1) (2010) 365 - 370. doi: 10.1021/cg900969n.

[31] M. M. Henricus, K. R. Fath, M. Z. Menzenski, I. A. Banerjee, Morphology controlled growth of chitosan-bound microtubes and a study of their biocompatibility and antibacterial activity, Macromol. Biosci. 9 (4) (2009) 317 - 325. doi:10.1002/mabi . 200800220.

[32] D. Yan, Y. Zhou, J. Hou, Supramolecular self-assembly of macroscopic tubes, Science 303 (5654) (2004) 65-67. doi:10.1126/science.1090763.

[33] G. J. T. Cooper, L. Cronin, Real-time direction control of self fabricating polyoxometalate-based microtubes, J. Am. Chem. Soc. 131 (24) (2009) 8368 - 8369. doi:10.1021/ja902684b.

[34] F. A. Ponce, D. P. Bour, Nitride-based semiconductors for blue and green light-emitting devices, Nature 386 (1997) 351 - 359. doi:10.1038/ $386351 \mathrm{a} 0$.

[35] S. Nakamura, The roles of structural imperfections in InGaN-based blue light-emitting diodes and laser diodes, Science 281 (5379) (1998) 956 961. doi:10.1126/science.281.5379.956.

[36] Y. C. Lan, X. L. Chen, Y. P. Xu, Y. G. Cao, F. Huang, Syntheses and structure of nanocrystalline gallium nitride obtained from ammonothermal method using lithium metal as mineralizator, Mater. Res. Bull. 35 (14 15) (2000) 2325 - 2330. doi:10.1016/S0025-5408(00)00447-5. 
[37] S. Li, A. Waag, GaN based nanorods for solid state lighting, J. Appl. Phys. 111 (7) (2012) 071101/1 - 071101/23. doi:10.1063/1.3694674.

[38] J. Y. Li, X. L. Chen, Z. Y. Qiao, Y. G. Cao, Y. C. Lan, Formation of GaN nanorods by a sublimation method, J. Cryst. Growth $213(3-4)$ (2000) 408 - 410. doi:10.1016/S0022-0248(00)00390-0.

[39] X. Chen, J. Li, Y. Cao, Y. Lan, H. Li, M. He, C. Wang, Z. Zhang, Z. Qiao, Straight and smooth GaN nanowires, Adv. Mater. 12 (19) (2000) 1432 1434. doi:10.1002/1521-4095(200010)12:19<1432: :AID-ADMA1432>3. $0 . \mathrm{CO} ; 2-\mathrm{X}$.

[40] X. L. Chen, Y. C. Lan, J. Y. Li, Y. G. Cao, M. He, Radial growth dynamics of nanowires, J. Cryst. Growth 222 (3) (2001) 586 - 590. doi:10.1016/ S0022-0248(00) 00971-4.

[41] T. Kuykendall, P. Pauzauskie, S. Lee, Y. Zhang, J. Goldberger, P. Yang, Metalorganic chemical vapor deposition route to GaN nanowires with triangular cross sections, Nano Lett. 3 (8) (2003) 1063 - 1066. doi: $10.1021 / \mathrm{nl} 034422 \mathrm{t}$.

[42] S. Y. Bae, H. W. Seo, J. Park, H. Yang, B. Kim, Porous GaN nanowires synthesized using thermal chemical vapor deposition, Chem. Phys. Lett. $376(3-4)(2003) 445$ - 451. doi:10.1016/S0009-2614(03) 00955-2.

[43] J. Goldberger, R. He, Y. Zhang, S. Lee, H. Yan, H.-J. Choi, P. Yang, Single-crystal gallium nitride nanotubes, Nature 422 (6932) (2003) 599 602. doi:10.1038/nature01551.

[44] M. He, I. Minus, P. Zhou, S. N. Mohammed, J. B. Halpern, R. Jacobs, W. L. Sarney, L. Salamanca-Riba, R. D. Vispute, Growth of large-scale GaN nanowires and tubes by direct reaction of $\mathrm{Ga}$ with $\mathrm{NH}_{3}$, Appl. Phys. Lett. 77 (23) (2000) 3731 - 3733. doi:10.1063/1.1329863. 
[45] J. Q. Hu, Y. Bando, J. H. Zhan, F. F. Xu, T. Sekiguchi, D. Golberg, Growth

[51] R. E. Liesegang, Ueber einige eigenschaften von gallerten, Naturwiss. Wochenschr. 11 (30) (1896) 353 - 362.

[52] M. Sacilotti, L. Imhoff, S. Bourgeois, C. Dumas, J. Decobert, P. Baldeck, I. Colombier, MOVPE growth of Ga 3D structures for fabrication of GaN of single-crystalline cubic GaN nanotubes with rectangular cross-sections, Adv. Mater. 16 (16) (2004) 1465 - 1468. doi:10.1002/adma. 200400016.

[46] L.-W. Yin, Y. Bando, M.-S. Li, D. Golberg, Growth of semiconducting GaN hollow spheres and nanotubes with very thin shells via a controllable liquid gallium-gas interface chemical reaction, Small 1 (11) (2005) $1094-$ 1099. doi:10.1002/smll.200500168.

[47] Y. Lan, H. Wang, F. Lin, Y. Lu, Y. Li, Y. Liu, J. Bao, Z. Ren, M. Crimp, Nanoporous gallium nitride square microtubes, J. Mater. Sci. 48 (21) (2013) 7703 - 7707. doi:10.1007/s10853-013-7590-4.

[48] X. L. Chen, Y. C. Lan, J. K. Liang, X. R. Cheng, Y. P. Xu, T. Xu, P. Z. Jiang, K. Q. Lu, Structure and heat capacity of wurtzite GaN from 113 to 1073 K, Chin. Phys. Lett. 16 (2) (1999) 107 - 108. doi:10.1088/ 0256-307X/16/2/011.

[49] V. Y. Davydov, Y. E. Kitaev, I. N. Goncharuk, A. N. Smirnov, J. Graul, O. Semchinova, D. Uffmann, M. B. Smirnov, A. P. Mirgorodsky, R. A. and AlN, Phys. Rev. B 58 (1998) 12899 - 12907. doi:10.1103/PhysRevB. 58.12899 .

[50] H. Siegle, G. Kaczmarczyk, L. Filippidis, A. P. Litvinchuk, A. Hoffmann, C. Thomsen, Zone-boundary phonons in hexagonal and cubic GaN, Phys. Rev. B 55 (1997) 7000 - 7004. doi:10.1103/PhysRevB.55.7000.

materials, J. Cryst. Growth $261(2-3)(2004) 253$ - 258. doi:10.1016/ j.jcrysgro.2003.11.065. 
[53] Z. W. Pan, Z. R. Dai, C. Ma, Z. L. Wang, Molten gallium as a catalyst for the large-scale growth of highly aligned silica nanowires, J. Am. Chem. Soc. 124 (8) (2002) 1817 - 1822. doi:10.1021/ja017284n.

[54] I. Lagzi, B. Kowalczyk, B. A. Grzybowski, Liesegang rings engineered from charged nanoparticles, J. Am. Chem. Soc. 132 (1) (2010) 58 - 60. doi: 10.1021/ja906890v.

[55] E. L. Spotz, J. O. Hirschfelder, Liesegang ring formation arising from diffusion of ammonia and hydrogen chlorine gases through air, J. Chem. Phys. 19 (9) (1951) 1215 - 1215. doi:10.1063/1.1748519.

[56] C. F. Goodeve, A. S. Eastman, A. Dooley, The reaction between sulphur trioxide and water vapours and a new periodic phenomenon, Trans. Faraday Soc. 30 (1934) 1127 - 1133. doi:10.1039/TF9343001127.

[57] L. V. Govor, G. Reiter, G. H. Bauer, J. Parisi, Nanoparticle ring formation in evaporating micron-size droplets, Appl. Phys. Lett. 84 (23) (2004) 4774 - 4776. doi:10.1063/1.1759378. 